\title{
LA ETNOBOTÁNICA MOQOIT INÉDITA DE RAÚL MARTÍNEZ CROVETTO II: DESCRIPCIÓN, ACTUALIZACIÓN Y ANÁLISIS DE LOS USOS DE LAS PLANTAS
}

\author{
GUSTAVO F. SCARPA ${ }^{1} \&$ CINTIA N. ROSSO ${ }^{2}$
}

\begin{abstract}
Summary: Scarpa, G. F. \& C. N. Rosso. 2014. The unpublished Raúl Martínez Crovetto's moqoits ethnobotany II: Description, updates and plants uses analysis. Bonplandia 23(2): 133141.

We have found unpublished and valuable moqoit ethnobotany data at the IBONE (Instituto de Botánica del Nordeste), gathered by Martínez Crovetto in the 60s at Chaco province. In this paper, we aim to systematize and disseminate those historical records, put them into their precise socio-cultural and spatio-temporal context, and finally, make a plant uses analysis. To achieve this goal, we use the methodology of "historical ethnobotany", that considers past data as a primary information source. Four hundred (400) ethnobotany data referring to 231 species were recorded. Ninety five percent (95\%) of them were native plants. Most used plants species were Prosopis alba, Copernicia alba, Zea mays, P. nigra, Bromelia serra and Schinus fasciculatus. Almost half of uses (49\%) were of medicinal nature; $23 \%$ alimentary and the remaining categories showed less than $5 \%$ of data each one of them. All this information becomes one of the most important background about moqoit ethnobotany that has been published so far.
\end{abstract}

Key words: Chaco, historical ethnobotany, mocoví, plant uses.

Resumen: Scarpa, G. F. \& C. N. Rosso. 2014. La etnobotánica moqoit inédita de Raúl Martínez Crovetto II: Descripción, actualización y análisis de usos de las plantas. Bonplandia 23(2): 133141.

En el Instituto de Botánica del Nordeste (IBONE) se hallaron valiosos datos inéditos sobre la etnobotánica moqoit que fueron documentados en la década de 1960 en la provincia del Chaco por Raúl Martínez Crovetto. Se propone sistematizar y difundir esta información histórica, contextualizarla espacio-temporal y socioculturalmente y analizar en términos generales los usos asignados a las plantas. Se emplea la metodología propia de la "etnobotánica histórica" que considera a los datos del pasado como una fuente de información primaria. Se registró un total de 400 datos etnobotánicos asignados a 231 especies. El 95\% de las plantas resultaron nativas. Las especies con mayor cantidad de usos registrados por Martínez Crovetto fueron $P$. alba, Copernicia alba, Zea mays, P. nigra, B. serra y Schinus fasciculatus. El $49 \%$ de aplicaciones fueron medicinales, el $23 \%$ alimenticias y el resto de las categorías utilitarias agrupaba menos del $5 \%$ de los datos. Esta valiosa información representa uno de los antecedentes más importantes sobre la etnobotánica moqoit publicados hasta el momento.

Palabras clave: Chaco, etnobotánica histórica, mocoví, usos vegetales.

${ }^{1}$ Investigador Adjunto CONICET, Museo Argentino de Ciencias Naturales "Bernardino Rivadavia"-CONICET. Angel Gallardo 470, 2º piso (C1405DJR) C.A.B.A. gscarpa@macn.gov.ar.

2 Becaria posdoctoral del Centro de Estudios Farmacológicos y Botánicos del CONICET. Paraguay 2155, piso $16^{\circ}$ (1121), C.A.B.A. cintia_rosso@yahoo.com.ar 


\section{Introducción}

Durante el desarrollo de una investigación etnobotánica en curso sobre la etnia moqoit (mocoví) los autores tuvimos la oportunidad de encontrar en la biblioteca del Instituto de Botánica del Nordeste (IBONE) (Corrientes, Argentina) una cuantiosa información inédita sobre esta temática que fuera recabada durante 1967 en el sudoeste de la provincia del Chaco por el Ing. Agr. Raúl Martínez Crovetto. La misma abarcaba numerosos datos fitonímicos y utilitarios, así como significados varios de las plantas. Debido al volumen de las informaciones compiladas y a los análisis que sobre ellas se han realizado, decidimos publicar por separado ambas contribuciones. La primera de ellas, titulada "La etnobotánica moqoit inédita de Raúl Martínez Crovetto I: Descripción, actualización y análisis de la nomenclatura indígena" (Scarpa \& Rosso, 2014), está dedicada a la fitonimia moqoit y constituye el antecedente directo de este trabajo.

Entre los antecedentes históricos sobre la etnobotánica moqoit figuran los del sacerdote jesuita Florián Paucke (1942-1944) (siglo XVIII), además de escasos datos publicados por Martínez Crovetto (1967, 1968a, 1968b, 1968c, 1970) y por Zapata Gollán (1945). En su artículo titulado "Introducción a la etnobotánica del Nordeste Argentino", Martínez Crovetto (1968b) refiere con fines comparativos la cantidad de "conocimientos florísticos" (especies nombradas) y "fitotecnológicos" (usos medicinales, alimenticios, ceremoniales, instrumentales, ictiotóxicos, etc.) recopilados entre los mocovíes, guaranímbyá, toba-takshík y vilela. Allí solo indica que los mocovíes utilizan un total de 227 especies vegetales principalmente como medicinas (55\%) y como alimentos (32\%), sin especificar las identidades de las plantas usadas ni las aplicaciones específicas de cada una de ellas, así como tampoco su fitonimia.

En la actualidad, a excepción del estudio de Rosso (2010), sobre un aspecto de su fitonimia, el de Scarpa \& Rosso (2011), que analiza la significación del "coro" (Nicotiana paa Mart. Crov.), y el de Rosso \& Scarpa (2012) sobre los datos etnobotánicos en la obra de Paucke, no han sido publicados otros trabajos acerca de la etnobotánica moqoit. Debido a la escasez de informaciones sobre esta temática, los datos inéditos considerados en este trabajo sobre las plantas útiles de este pueblo cobran una relevancia particular, ya que constituirían los registros etnobotánicos más exhaustivos hallados sobre esta etnia hasta la actualidad.

Raúl Martínez Crovetto (M.C.) fue profesor durante veinticuatro años de la cátedra de Botánica Sistemática y Fitogeografía en la Facultad de Ciencias Agrarias de la Universidad Nacional del Nordeste(UNNE) y es considerado pionero de las investigaciones etnobotánicas propiamente dichas en Argentina (Arenas, 1992). Además de la precisión botánica y etnográfica, de estos datos inéditos, el hecho de que hayan sido registrados hace ya más de 45 años le confieren una importancia adicional en vistas del cambio cultural sobrevenido en esta etnia (que habría determinado el olvido y/o la pérdida de prácticas y conocimientos), así como de la alta tasa de deforestación sufrida en todo el Chaco Oriental desde esa época a la actualidad ${ }^{3}$. Por ello, muchos de los datos recopilados por M.C. resultan imposibles de volver a registrarse a campo en la actualidad. La validez y relevancia de otros datos inéditos de este autor ya fue destacada en otras obras donde algunos de ellos han sido publicados (Viegas Barros, 1995; Pirondo \& Keller, 2012).

Los objetivos de este trabajo son sistematizar la información histórica inédita recopilada por los autores, contextualizarla desde las perspectivas espacio-temporal y sociocultural, discutir las identificaciones botánicas de los taxa referidos y analizar los conocimientos y usos asignados a las plantas, en particular los datos sobre etnobotánica médica y alimentaria de las mismas.

\footnotetext{
${ }^{3}$ A pesar de que la zona donde el citado investigador registró sus datos es históricamente agrícola, antes con cultivos de algodón preferentemente, es en las últimas décadas cuando se intensifica especialmente la deforestación del bosque chaqueño relictual de la zona, debido al aumento de la superficie sembrada y a la diversificación de los cultivos practicados (soja, girasol, maíz, etc.).
} 


\section{Materiales y Métodos}

Los materiales empleados provienen de manuscritos inéditos del propio M.C. que se encontraron en la Biblioteca del Instituto de Botánica del Nordeste (IBONE) de la Facultad de Ciencias Agrarias (CONICET-UNNE) en la ciudad de Corrientes, Argentina. Los mismos se hallaron en una caja de cartón rotulada: "Caja Mocoví, Ms. Martínez Crovetto", ordenados en tres paquetes de fichas atadas y etiquetados de la siguiente manera: 1. "Mocobí - latino. 327 nombres (10/1/67)"; 2. "Latino - Mocoví. 475 spp. (9/9/67)" y 3. "Mocovíes. 11/10/67”. El primero de ellos lo conformaban 323 fichas inicializadas en su borde superior izquierdo con uno o más fitónimos en lengua moqoit, se hallaban ordenadas alfabéticamente según este criterio y en renglones subsiguientes figuraba el o los nombres científicos a los que correspondían dicho/s nombre/s vernáculo/s. El segundo paquete contaba con 475 fichas inicializadas y ordenadas alfabéticamente por nombre científico y en renglones subsiguientes el o los fitónimos moqoit registrados para dicha especie. El tercer paquete, correspondiente a los datos que se ordenan y analizan en este trabajo, incluía 227 fichas ordenadas alfabéticamente por nombre científico sobre cada una de las cuales figuraba una somera descripción de uno o más datos etnobotánicos recabados de primera mano entre pobladores moqoit.

Además de estos paquetes, la caja citada conservaba una carpeta con páginas mecanografiadas cuyo contenido se dividía en dos partes: una titulada "Índice de las especies útiles entre los mocovíes del Chaco" y otra "Arquitectura de la fitonimia mocoví". La primera de ellas consistía en un listado de taxones botánicos, y de fitónimos moqoit en menor medida, ordenados según tipos y subtipos de usos de las plantas, tal como será detallado en los Resultados.

La metodología de análisis empleada en este trabajo es aquella que considera a los datos del pasado como si fueran una fuente de información primaria, a los cuales se les aplica la metodología propia de la etnobotánica histórica (Medeiros, 2009; Rosso, 2012 y Rosso \& Scarpa, 2012).
En vistas a los avances registrados en las disciplinas botánica y etnobotánica, y a los cambios culturales y ambientales operados desde la época en que fueron tomados los datos a la actualidad, todo abordaje de este tipo debería trascender la simple editorialización y ordenamiento de la información histórica, inédita o publicada. Por ello consideramos que ésta debe ser contextualizada desde el punto de vista espacio-temporal y sociocultural, para luego revisar las identificaciones botánicas de los taxa referidos. Respecto a esto último, se procedió a verificar la validez del binomio, su inclusión como "aceptados" para la flora argentina, su distribución en la provincia del Chaco y su actualización en caso de su condición de "sinónimo", según las bases de datos "Flora del Cono Sur" del Instituto de Botánica Darwinion (http://www2.darwin. edu.ar/Proyectos/FloraArgentina/FA.asp\#) y "Tropicos" del Missouri Botanical Garden (http://www.tropicos.org/), ambas de actualización permanente (a excepción de las tres especies de hongos cuyos nombres científicos no fueron actualizados).

\section{Resultados}

\section{Contextualización espacio-temporal y sociocultural}

Respecto a los asentamientos moqoit cuyos representantes brindaron estos datos, todas las publicaciones de M.C. al respecto coinciden en afirmar que se trata de aquellos ubicados en el centro-sur de la provincia del Chaco, cercanos a las localidades de Charata (Colonia Gral. Necochea), Villa Ángela y Villa Berthet. A este respecto, es especialmente ilustrativa su publicación titulada "Estado actual de las tribus mocovíes del Chaco", donde detalla su localización, así como su etnohistoria reciente en los siguientes términos: "A principios de este siglo $[\mathrm{XX}]$, un grupo de indios mocovíes, compuesto por unos 500 individuos de ambos sexos, dejó la región santafecina de San Javier, lugar de su origen y se internaron entre los montes casi inexplorados del centro de la actual provincia del Chaco. Durante más de 20 años vivieron como nómades, hasta que, hacia el año 1922, decidieron establecerse 
definitivamente en las vecindades de la joven localidad de Charata, mientras hacían venir de Santa $\mathrm{Fe}$ a sus parientes y amigos, aprovechando el auge económico promovido por el cultivo del algodón" (Martínez Crovetto, 1968b: 1). A pesar de que este desplazamiento poblacional sería de larga data, los autores pudimos constatar en una de las localidades referidas (Colonia Gral. Necochea) la presencia de descendientes originarios de las riberas del río Paraná (donde se ubica la localidad citada de San Javier).

Según la contrastación realizada entre los datos referidos en los manuscritos hallados y los publicados sobre los moqoit por Martínez Crovetto (1967, 1968a, 1968b, 1968c y 1970), podemos colegir que aquellos habrían sido registrados a campo, al menos, desde el año 1966. Esto deducimos en función de la fecha de su primer trabajo publicado sobre esta etnia y del hecho de que, en enero de 1967, ya declaraba haber registrado un total de 327 de sus fitónimos. Asimismo, las informaciones habrían sido registradas hasta el año 1967 , considerando la última fecha de los datos declarados en sus fichas y que los demás trabajos fueron publicados durante del primer semestre de 1968.

\section{Identificaciones botánicas de los taxa referidos}

El análisis de las identificaciones botánicas históricas de las plantas cuyos usos analizamos en esta contribución, fue realizado previamente en el trabajo ya citado de Scarpa \& Rosso (2014), por lo que para ello remitimos al lector a dicha publicación. Las únicas referencias que pudimos hallar respecto de la existencia de material vegetal colectado por el autor, son ciertas anotaciones del tipo "M678" que figuran en varias de las fichas correspondientes a taxones que M.C. no pudo identificar o en aquellas que contienen determinaciones a nivel de género. Según el Ing. Krapovickas (com. pers.) este código indica el número de muestra herborizada por dicho autor, quien la habría depositado en el Herbario CTES (IBONE). Esta observación resulta congruente con lo señalado por Arenas (1992) respecto al hábito de M.C. de coleccionar a campo solo aquellas plantas que le resultaban desconocidas.
Por otro lado, en fichas correspondientes a especies de Fabaceae, hay señales de que M.C. habría consultado a especialistas para su determinación, como en la que consigna: "A.B." y en otras consigna directamente "según Burkart". Por ello deducimos que aquellas iniciales corresponderían al botánico Arturo Burkart, máximo referente argentino para tal familia botánica.

\section{Conocimientos y usos asignados a las plantas}

3.A. Generalidades. Según MartínezCrovetto (1968b) la cantidad de especies vegetales -y hongos- que los moqoit conocían ascendía a 475, de las cuales utilizaban un total de 227. Así, la relación entre el número de especies utilizadas y las conocidas (o nombradas) hasta el momento en que M.C. realizó este conteo, era de tan solo el 48\% (proporción muy baja para cualquier investigación etnobotánica , ver Discusión). El hecho de que el número de plantas utilizadas que fuera publicado en dicha obra coincida con el número de fichas encontradas por nosotros en el tercer paquete rotulado "Mocovíes", reafirma que la autoría de todas ellas y la información que contienen corresponden indudablemente a Raúl Martínez Crovetto.

El "Índice de las especies útiles entre los mocovíes del Chaco" presente en la carpeta mecanografiada ya mencionada (ver Materiales y Métodos), consiste en un listado de taxones botánicos, y de fitónimos moqoit en menor medida, agrupados en función de las siguientes categorías y subcategorías de uso de las plantas: "Agua para beber" (1 taxón); "Alimenticias" (72); "Armas" (11); "Ataduras" (4); "Bastos" (1); "Bebidas" (8); "Colchones (rellenos)"(1); "Construcción" cercos y techados" (14); "Escobas" (2); "Esteras" (2); "Estribos" (1); "Extracción de miel" (1); "Fuego (para encender) (2)"; "Herramientas y mangos" (1); "Fumatorios" (2); "Instrumentos musicales" (2); "Leñas" (4); "Medicinales" (125); "Veterinaria" (5); "Pipas para fumar" (2); "Recipientes, bateas y morteros" (3); "Sombreros" (1); "Supersticiones y amuletos" (12); "Textiles" (2); "Tintóreas" (4); "Tóxicas" (1); "Yesca" (2) y "Para ennegrecer cerámica" (1 taxa). 
A diferencia de las demás categorías, las "Medicinales" y "Alimenticias" presentan una subcategorización especial, la cual se debe a la gran diversidad de datos etnobotánicos que estas involucran ( $87 \%$ del total). En este sentido, M.C. agrupa los taxones botánicos según las siguientes subcategorías de uso medicinal: "aires y pasmo"; "alumbramiento"; antiblenorrágicos; "anticatarrales"; anticaspa; anticonceptivos; antidiarreicos; antidiabéticos; antigripal; antihemorrágico y hemostático, antirreumático; "antivomitivo"; astringentes y antisépticos; "bocio"; "cefalalgias"; "conjuntivitis"; "coqueluche"; "conceptivos"; "corazón"; "depurativo para la sangre"; diurético; "dolores corporales"; "dolor de muelas"; "dolor de oídos"; "dolor de garganta"; "dolor de riñón"; "dolor de vejiga"; "dolores de vientre"; "dolor estomacal"; "dolor intestinal"; "embriaguez"; estomacal y digestivo; febrífugos, "fiebres intermitentes"; "fortalecer sangre"; "forúnculos y granos"; "fracturas y recalcaduras", galactógenos; "geofagia"; "golpes"; hepáticos, "hinchazones"; "insolación"; laxantes y purgantes; "menstruación dolorosa"; miasis ("queresa"); "mejorar visión"; "mordedura víbora"; "paperas"; "quemaduras"; "remedio fresco"; "riñón"; sedante; "vejiga"; "vegetación de la nariz [sic]".

Asimismo, los taxones botánicos referidos a la categoría "Alimenticias" se hallan ordenados en las siguientes subcategorías de uso: "condimentos"; "frutas"; "médulas"; "partes aéreas"; "rizomas y bulbos", "semillas" y "tubérculos".

3.B. Análisis de los datos. En un artículo aparte de este volumen de la revista (Martínez Crovetto, 2014) se detallan los usos de las plantas consignados por M.C. Como resultado del análisis de todas las fuentes donde M.C. indica "usos" de las plantas (fichas y carpetas), pudimos registrar un total de 231 especies "útiles" (4 más que lo contabilizado por el autor) sobre las cuales se refirieron 400 datos etnobotánicos. El 95\% de las plantas resultaron nativas (221 especies) y solo 10 exóticas, la mayoría de ellas cultivadas.

Se procedió a comparar los datos registrados en ambas fuentes, es decir, entre los referidos en las fichas y aquellos enlistados en la carpeta mecanografiada, para cada una de las especies en cada una de las categorías y subcategorías señaladas. De los 195 usos señalados en las fichas como "medicinales" se pudo constatar que 23 de ellos (12\%) no se encontraron en los listados correspondientes a la carpeta mencionada. Por otro lado, se encontraron dos usos que no se hallaron en las fichas pero sí en la carpeta, razón por la cual no figura la parte utilizada (en un solo caso figuran especies distintas en una y otra fuente para el mismo uso).

Las especies con mayor cantidad de usos registrados por M.C. fueron Prosopis alba (9 datos), Copernicia alba y Zea mays (con 6 datos cada una), y P. nigra, B. serra y Schinus fasciculatus (con 5 datos cada una). El 49\% de aplicaciones (195 datos) fueron medicinales; el 23\% (93 datos) alimenticias y el resto de las categorías utilitarias $(28 \%, 112$ datos) agrupaba menos del 5\% de los datos cada una de ellas, tal cual se grafica en la Fig. 1.

Los usos medicinales (195) fueron asignados a 125 especies vegetales, las más importantes de las cuales fueron Buddleja brasiliensis, Heimia salicifolia y Talinum racemosum (con 4 usos cada una de ellas). Las principales subcategorías de uso medicinal correspondieron al aparato digestivo $(20 \%$, 38 datos), al aparato reproductor $(16 \%, 31$ datos), al aparato respiratorio $(12,5 \%, 24$ datos) y a trastornos con manifestaciones en la superficie corporal (10,5\%, 20 datos). A nivel de las aplicaciones específicas, las plantas empleadas como digestivo estomacal (14 datos), antitusivas (13), febrífugas (10) y contra la conjuntivitis (9 datos) resultaron las más destacadas.

Los usos asignados a la categoría "Alimenticias" (93) fueron asignados a 70 especies vegetales, 64 de las cuales eran nativas. Las más destacadas según las preparaciones culinarias en las que participaban fueron Prosopis alba (6 datos), Copernicia alba (4 datos) y P. nigra (4 datos). En la mayoría de las ocasiones $(59,63 \%)$ las partes vegetales se consumen crudas y en menor medida (17 datos, 18\%) cocidas (hervidas o asadas). Se registraron además 13 datos en la que las partes vegetales se consumen en forma de bebidas, 7 como harinas y 6 como condimentos. 


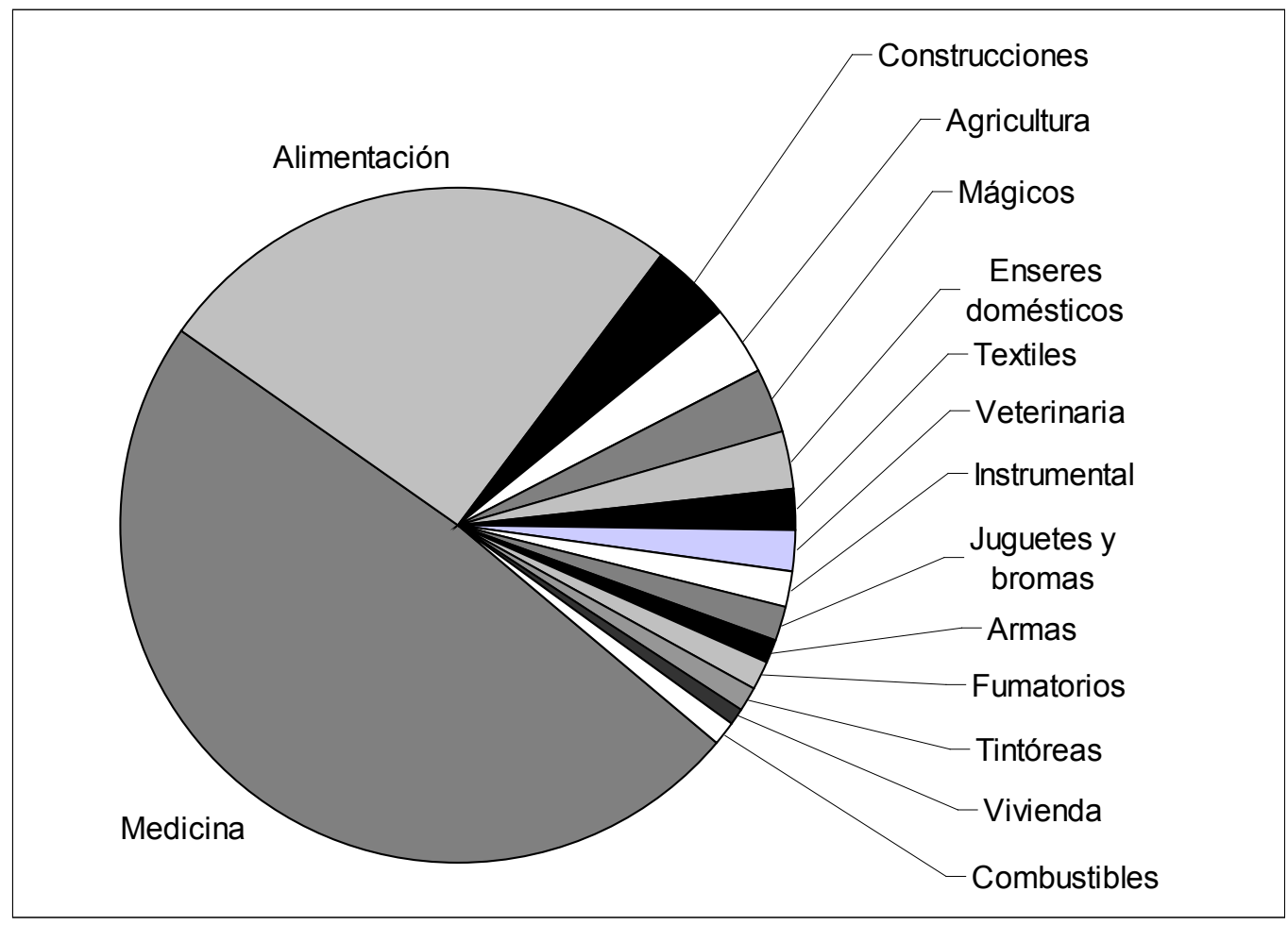

Fig. 1. Categorización del uso de las plantas.

Los datos agrupados en otras categorías de usos (112) fueron referidos para 88 especies vegetales. Las más importantes son las empleadas en construcciones (15 datos), agricultura (13), con fines mágicos (12), en enseres domésticos (9), veterinaria (8), textiles (8), instrumental (7), armas (6), con fines lúdicos (6) y como fumatorios (5).
Por último, se debe destacar que los niveles de detalle referidos en las fichas sobre los usos de cada una de las plantas resultaron ciertamente heterogéneos, ya que figuran tanto anotaciones tales como "su fruto es comestible", así como descripciones más o menos detalladas (todas ellas se reproducen como nota al pie $)^{4}$.

${ }^{4}$ Capparis cynophallophora: "Comen los porotos llamados "teguek" hervidos. Se hierven con la vaina en dos aguas diferentes con sal y después se pelan. Se consumen estofados, guisados o simplemente empastados con grasa de caballo, vaca o ñandú derretida. Se consumen tiernos o maduros y solos o incluyendo la chaucha. Se prefieren tiernos. Sin los dos hervores resultan amargos.". Desmanthus chacoense: "La Dec. de las raíces en un litro de agua le dan de beber a las mujeres ya sea directamente o en el mate sin que sospechen a aquellos hombres que hacen mala vida, es decir, que son bebedores, pendencieros, mujeriegos, etc. para que mejoren.”. Prosopis alba: "Comen las vainas crudas o asadas, o bien hacen harinas llamadas "ne'naGa". Para ello se ponen las vainas al sol, siendo suficiente un día si el tiempo es bueno. En caso contrario las ponen sobre un encatrado de palos con fuego lento suave debajo y se remueven convenientemente. Luego se pisan en un mortero grande. Las harinas se consumen desleídas en agua, lo cual se llama "nekená". También preparan con ella una especie de patay, para lo cual, la harina se cierne y se pone en un cuero de pierna de avestruz y se atan ambos extremos y se deja colgado de uno a dos meses, con lo que la harina se pone dura y consistente. La harina se emplea también para preparar panes de mistol. Algunas le sirven para preparar chicha que se llama "kapaGa", la cual se fermenta en bateas largas llamadas "netá natá naki", hecha con un trozo de tronco de algarrobo blanco o negro. Con los troncos ahuecados a fuego fabrican morteros. También fabrican pipas llamadas "naserak nakí" con su madera. Salicornia sp. "Se incinera su parte aérea. Con las cenizas y agua se amasan y forman bolitas. Estas, una vez solidificadas, se raspan o se pulverizan trozos con los dedos y espolvorean sobre las comidas." 


\section{Discusión y Conclusiones}

La proporción de plantas útiles registrada por M.C. (48\%) para los moqoit resulta extremadamente baja si la comparamos con la que frecuentemente se registra en investigaciones etnobotánicas. En el antecedente directo a este trabajo (Scarpa \& Rosso, 2014) ya se advierte una evidente desproporción entre la exhaustividad de nombres moqoit consignados y la relativa escasez de datos sobre usos y significaciones aquí registrados. Así lo sugieren algunos trabajos etnobotánicos sobre pueblos de la región en los cuales la proporción de plantas utilizadas no suele ser inferior al $80 \%$ del total conocido, tal como registra Filipov (1996) para los pilagá del Chaco Subhúmedo (81\%), Scarpa (2012, obs. pers.) con los criollos (85\%) y con los chorote del Chaco Semiárido norte $(81 \%)$ respectivamente. El bajo cociente antes referido corresponde típicamente a resultados parciales propios de cualquier investigación etnobotánica, lo que indicaría que M.C. se habría hallado promediando su trabajo de campo.

Dado que la medicina de los indígenas chaquenses es eminentemente de tipo chamánica, se ha registrado que la participación de plantas como medio terapéutico propiamente dicho suele ser escasa en proporción a los usos o significaciones totales hallados (p. ej. 15\% entre chorotes), a pesar de las adquisiciones culturales comprobadas en el ámbito de su etnobotánica médica (Arenas, 2000, 2009; Scarpa, 2009, 2013). Sin embargo, los resultados consignados por M.C. indican que los usos medicinales son los más conspicuos de todos (47\% de los usos totales), en el mismo orden que lo registrado entre los criollos del Chaco Semiárido norte (41\%) (Scarpa, 2012). A diferencia de los resultados anteriores, Martínez (2008) registró entre los qom-bermejeños de la provincia del Chaco 893 usos medicinales de las plantas, lo cual representaría una proporción igualmente mayoritaria de sus datos etnobotánicos totales, tal como se registra en este trabajo. Coincidimos con este autor respecto a que estas diferencias con las demás etnias chaquenses podrían ser explicadas como consecuencia de la adquisición de numerosos conocimientos y prácticas herbolarias medicinales de sus vecinos criollos (el hecho de que los qom y los moqoit compartan su residencia en el Chaco Húmedo y su pertenencia a la misma familia lingüística, añadiría mayores coincidencias a los resultados obtenidos).

La cantidad de aplicaciones medicinales asignadas al aparato digestivo como principal subcategoría de uso terapéutico, resulta del todo idéntica a lo registrado para la etnobotánica médica de grupos criollos del Chaco y de otras zonas de Argentina (Scarpa, 2002; Martínez, 2010; Muiño, 2010). Asimismo, el registro de aplicaciones terapéuticas (tales como antidiabéticos, para "limpiar la sangre" o contra el "pasmo") y alimenticias específicas de las plantas (como para preparar el "bolanchao" o el "patay"), constituyen otros ejemplos contundentes de la inclusión temprana de categorías gnoseológicas típicas de la etnomedicina y de la alimentación criolla al acervo cultural de los indígenas moqoit.

Elnúmerototaldeplantas nativas alimenticias registradas entre los moqoit (64) se ubica dentro del rango promedio $(60+/-12)$ estimado por Scarpa (2009) en su estudio comparativo de estas plantas para 10 grupos étnicos del Gran Chaco. La proporción entre la cantidad de preparaciones culinarias y diversidad de especies nativas alimenticias de 1:4 registrada por M.C., resulta significativamente menor en comparación al promedio de 2,0 +/0,16 estimado para cinco grupos indígenas chaquenses por Arenas \& Scarpa (2007). Consideramos que esto también sería atribuible a la condición preliminar del trabajo de campo de M.C. ya mencionada. A diferencia de lo registrado para el ámbito de la etnobotánica médica, inferimos que los usos alimenticios de las plantas estarían subestimados debido al cambio cultural operado, tal como sugieren las observaciones que en dicha época fueran registradas por M.C. en estos términos: "Las [plantas] alimenticias principalmente toman ahora un valor secundario, pasando a ser una golosina para los chicos, o terminando en el desuso" (Martínez Crovetto, 1970).

Las especies vegetales sobre las que se registrómayor cantidad de usos o significaciones resultaron semejantes a las halladas entre otras 
etnias chaquenses (Prosopis spp.; Copernicia $a l b a$, etc.), a excepción de la escasa utilización de especies tales como Geoffroea decorticans, Ziziphus mistol o Acacia spp. y de la alta cantidad de datos registrados para Schinus fasciculatus. Entre los usos registrados por M.C. resulta novedosa la inclusión de hojas de Nicotiana longiflora como comestibles en forma de ensaladas (primera cita para el país) y de las fibras de Dyckia sp. como textiles. A diferencia del primer uso, el de Dyckia sp. como textil ya fue citado para los moqoit en la obra de Rosso \& Scarpa (2012) sobre la base de una entidad botánica citada por el sacerdote Paucke únicamente por su nombre vulgar moqoit (cuya correspondencia con el taxón citado es confirmado por M.C. aquí). Debe destacarse que otros autores niegan este uso textil de Dyckia sp. entre etnias chaquenses en general (Arenas, 1997) o para los wichí en particular (Suárez \& Montani, 2010).

Según los antecedentes mencionados en la introducción, la gran cantidad de datos originales analizados en este artículo y compendiados a nombre de Raúl Martínez Crovetto en este número de Bonplandia por los autores, constituye sin lugar a dudas el antecedente más importante sobre la etnobotánica moqoit publicado hasta el momento.

\section{Agradecimientos}

Al Ing. Antonio Krapovickas y a las autoridades y bibliotecarios del Instituto de Botánica del Nordeste, por la amabilidad de habernos permitido acceder a los archivos de Raúl Martínez Crovetto, a Eugenia Suárez por sus valiosas sugerencias y a Macarena Perusset por su ayuda en la traducción del resumen.

\section{Bibliografía}

ARENAS, P. 1992. Raúl Nereo Martínez Crovetto (19241988), su contribución a la etnobotánica y a los estudios americanistas. Parodiana 5: 505-519.

. 1997. Las bromeliáceas textiles utilizadas por los indígenas del Gran Chaco. Parodiana 10: 113-139.

- 2000. Farmacopea y curación de enfermedades entre algunas etnias del Gran Chaco. En A. G.
Amat (comp.), Farmacobotánica y farmacognosia en Argentina 1980-1998, pp. 87- 118. Ediciones Culturales Argentinas, La Plata. 298 pp.

. 2009. Los estudios sobre medicina y farmacopea vernácula en el Gran Chaco. Rojasiana 8: 81-100.

\& G. F. SCARPA. 2007. Edible wild plants of the Chorote Indians, Gran Chaco, Argentina. Bot. J. Linn. Soc. 153: 73-85.

FILIPOV, A. 1996. Estudio etnobotánico de la recolección entre los Pilagá. Tesis Doctoral, Facultad de Ciencias Naturales y Museo de la Universidad Nacional de La Plata. $191 \mathrm{pp}$.

MARTÍNEZ, G. J. 2008. La farmacopea natural en la etnomedicina de los Tobas del Río Bermejito (Chaco, Argentina). Tesis Doctoral, Facultad de Ciencias Agropecuarias. Universidad Nacional de Córdoba. 248 pp.

. 2010. Las plantas en la medicina tradicional de las Sierras de Córdoba. Ediciones del Copista, Córdoba. $212 \mathrm{pp}$.

MARTÍNEZ CROVETTO, R. 1967. Contribución al estudio de la cerámica actual de los indios mocovíes del Chaco (República Argentina). Etnobiológica 1: 1-7.

—. 1968a. Viejos juegos de los indios mocovíes. Etnobiológica 2: 1-31.

1968b. Estado actual de las tribus mocovíes del Chaco (República Argentina). Etnobiológica 7: 1-33.

- 1968c. Introducción a la etnobotánica del Nordeste Argentino. Etnobiológica 11: 1-10.

- 1970. La etnobotánica de los grupos aborígenes del nordeste argentino. Bol. Soc. Argent. Bot. 11(Supl.): 211-215.

—. 2014. Algunos datos sobre etnobotánica mocoví. Bonplandia (este número).

MEDEIROS, N. H. 2009. Etnobotánica histórica: princípios e procedimentos. Sociedade Brasileira de Etnobiologia e Etnoecologia. Série Estudos e debates (Sociedade Brasileira de Etnobiologia e Etnoecologia) v. 6. PE: NUPEEA, Recife.

MUIÑO, W. A. 2010. El uso de las Plantas Silvestres por la Comunidad de Chos Malal (Provincia de La Pampa). Tesis Doctoral, Facultad de Ciencias Naturales y Museo, Universidad Nacional de La Plata. 276 pp.

PAUCKE, F. 1942-1944. Hacia allá y para acá. Una estadía entre los indios Mocobíes, 1749-1767. Volumen I, II y III. Universidad de Tucumán, Tucumán.

PIRONDO, A. \& H. A. KELLER. 2012. Raúl N. Martínez Crovetto: los albores de la etnobotánica en la Argentina. Introducción a Estudios Etnobotánicos V. Bonplandia 21: 101-107.

ROSSO, C. N. 2010. Compilación y análisis preliminar de la fitonimia de la flora leñosa de comunidades mocovíes del sudoeste chaqueño. En C. Messineo, G. F. Scarpa \& F. Tola (comps.), Léxico y 
categorización etnobiológica en grupos indígenas del Gran Chaco, pp. 251-271. Universidad Nacional de La Pampa, Santa Rosa.

2012. La etnobotánica de los grupos mocovíes de la reducción de San Javier, en el Gran Chaco, durante el siglo XVIII. Tesis Doctoral, Facultad de Filosofía y Letras, Universidad de Buenos Aires. 306 pp.

\& G. F. SCARPA. 2012. Identificaciones botánicas de las plantas empleadas entre los mocovíes en la reducción San Javier durante el siglo XVIII a partir de la obra de Florián Paucke, S. J. En P. Arenas (ed.), Etnobotánica en zonas áridas y semiáridas del Cono Sur de Sudamérica, pp. 45-70. Sigma, Buenos Aires.

SCARPA, G. F. 2002. Plantas empleadas contra trastornos del sistema digestivo en la medicina folk de los Criollos del Chaco Noroccidental argentino. Dominguezia 18: 36-50.

2009. Wild food plants used by the indigenous peoples of the South American Gran Chaco: A general synopsis and intercultural comparison. J. Appl. Bot. Food Qual. 83: 90-101.

. 2012. Las plantas en la vida de los criollos del oeste formoseño. Medicina, Ganadería, Alimentación y Viviendas Tradicionales. Rumbo Sur, Buenos Aires. 240 pp.

2013. Medicina indígena chorote. Continuidad y transformaciones históricas y culturales. Editorial Académica Española, Norderstedt. 158 pp.
— \& C. N. ROSSO. 2011. Etnobotánica del "coro" (Nicotiana paa, Solanaceae): Un tabaco silvestre poco conocido del extremo sur de Sudamérica. Bonplandia 20: 391-404.

—. 2014. La etnobotánica moqoit inédita de Raúl Martínez Crovetto I: Descripción, actualización y análisis de la nomenclatura indígena. Bol. Soc. Argent. Bot. 49 (en prensa).

SUÁREZ, M. E. \& R. M. MONTANI. 2010. Vernacular knowledge of Bromeliaceae species among the wichí people of the Gran Chaco, Argentina. J. Ethnob. 30: 265-288.

TROPICOS.ORG. 2009. Missouri Botanical Garden. Disponible en: http://www.tropicos.org. [Acceso: 28 April 2014]

VIEGAS BARRIOS, J. P. (ed.) 1995. Zoonimia y etnozoología de los Pilagá, Toba, Mocoví, Mataco y Vilela. Facultad de Filosofía y Letras de la Universidad de Buenos Aires, Buenos Aires. 188 pp.

ZAPATA GOLLÁN, A. 1945. Nomenclatura mocobí de animales y plantas. Boletín del Departamento de Estudios Etnográficos y Coloniales 1: 51-62. Santa Fe.

ZULOAGA, F. \& O. MORRONE. 2009. Flora del Cono Sur. Catálogo de las Plantas Vasculares. Instituto de Botánica "Darwinion", Buenos Aires. Disponible en: http://www2.darwin.edu.ar/Proyectos/ FloraArgentina/FA.asp. [Acceso: 28 April 2014].

Original recibido el 21 de agosto de 2014; aceptado el 30 de octubre de 2014. 
